\section{LA-UR- $94-3655$}

Title:

Author(s):

Submitted to:
Anisotropic High Temperature Superconductors as Variable Resistors and Switches
Heinrich J. Boenig, Mark A. Daugherty, Steven Fleshler, Martin P. Maley, Fred M. Mueller, F. Coyne Prenger, and J. Yates Coulter

1994 Applied Superconductivity Conferences

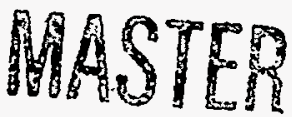

DISTRIBUTION OF THIS DOCUMENT IS UNLIMITED

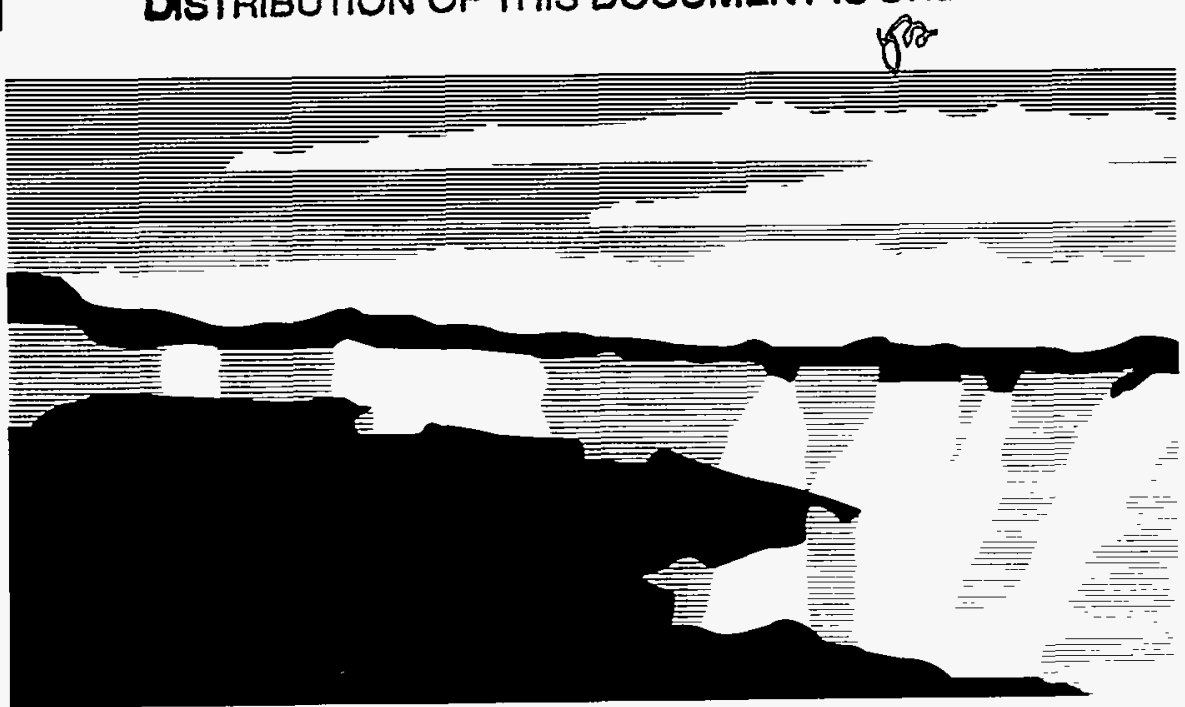

Los Alamos National Laboratory, an affirmative action/equal opportunity employer, is operated by the University of California for the U.S. Department of Energy under contract W-7405-ENG-36. By acceptance of this article, the publisher recognizes that the U.S. Government retains a nonexclusive, royalty-free license to publish or reproduce the published form of this contribution, or to allow others to do so, for U.S. Government purposes. The Los Alamos National Laboratory requests that the publisher identify this article as work performed under the auspices of the U.S. Department of Energy. 


\section{DISCLAIMER}

Portions of this document may be illegible in electronic image products. Images are produced from the best available original document. 


\title{
Anisotropic High Temperature Superconductors as Variable Resistors and Switches
}

\author{
Heinrich J. Boenig, Mark A. Daugherty, Steven Fleshler, Martin P. Maley, \\ Fred M. Mueller, F. Coyne Prenger, J. Yates Coulter
}

Los Alamos National Laboratory, Los Alamos, NM 87545

\begin{abstract}
Several anisotropic high temperature superconductors show critical current densities which are strongly dependent on the direction of an applied external magnetic field. The resistance of a sample can change by several orders of magnitude by applying a magnetic field. The potential for using the field dependent variable resistor or switch for applications in power systems is evaluated. Test results with small samples are presented. The requirements for large scale applications are outlined. The magnetic field triggering requirement, the frequency response of the device, use in $60 \mathrm{~Hz}$ ac circuits and heat transfer considerations are investigated. Several application examples are discussed. Use of the variable resistor as a fault current limiter, as a switching element in rectifier circuitry and as an improved dump resistor for a superconducting magnet is presented.
\end{abstract}

\section{INTRODUCTION}

The current carrying capability of both low temperature and high temperature superconductors decreases with the application of a magnetic field. In general, this feature is undesirable, because the current carrying capability and, therefore, the stability margin are lowered even by the self field of the current in the superconductor. Some anisotropic high temperature superconductors, in particular the bismuth and thallium based superconductors, show a resistance that is highly dependent on the amplitude and direction of the applied field[1]-[3]. The resistance change can be several orders of magnitude for a given conductor sample, when exposed to an external magnetic field along the crystallographic $\mathrm{c}$ axis compared to the no field condition. Although the field dependency of this special class of superconductors is undesirable in most applications, it appears that in some applications these unique characteristics might lead to innovative solutions.

\section{RESISTANCE-FIELD DEPENDENCE OF HTS WIRES}

Anisotropic HTS materials show a dependence of the critical current density, and therefore the resistivity, on the direction of the applied magnetic field. With reference to Fig. 1, if the magnetic field is parallel to the basal ab plane, the critical current density is little influenced by the applied

Manuscript received October 18, 1994

This work was conducted at the Los Alamos National Laboratory and supported by the United States Department of Energy, Office of Energy Management. external magnetic field. However, if the magnetic field is perpendicular to the ab plane, a steep exponential reduction with field in the critical current density is observed. By rotating a HTS wire sample along the a axis in a constant magnetic field, the voltage varies as a function of angle $\theta$, as shown in Fig. 2. The measurements were made at a temperature of $75 \mathrm{~K}$. The test sample was a $1 \mathrm{~cm}$ long BSCCO tape with a silver sheath. A constant current of 2.5 A flowed through the sample and the constant external magnetic field was 4000 Gauss. The measured voltage drop is directly proportional to the resistance of the sample because the current is constant. The resistance of the sample shows, to a first approximation, a sinusoidal dependence on the angle $\theta$, which is formed by the $c$ axis and the direction of the external field. The sample resistivity is the highest, when the field is parallel to the $c \operatorname{axis}(\theta=0)$.

While the voltage drop and resistance values of the samples shown in Fig. 2 are rather modest, larger values can be achieved with longer sample lengths. The V-I characteristics of a thallium based short sample is shown in Fig. 3 for a field of variable strength parallel to the $c$ axis. The sample, which is commercially available, is $10 \mathrm{~cm}$ long and is formed in a meander line fashion. The superconductor is T12212 on a Lanthanum Aluminate substrate. The sample has outside dimensions of $5 \mathrm{~mm}^{2}$. A detailed description of the sample is given in reference [4]. The figure clearly shows that, with increasing magnetic field, the critical current of the sample decreases. While the sample can carry a current of $0.4 \mathrm{~A}$ in the superconducting state with no background field, the current carrying capability is reduced to $0.1 \mathrm{~A}$ with an applied external field of 200 Gauss. The resistance of the sample in the flux flow state is limited by the resistivity of the sheath or substrate material. The resistivity of the sheath or substrate material should be high to achieve a large resistance ratio between the resistive and the superconducting state.

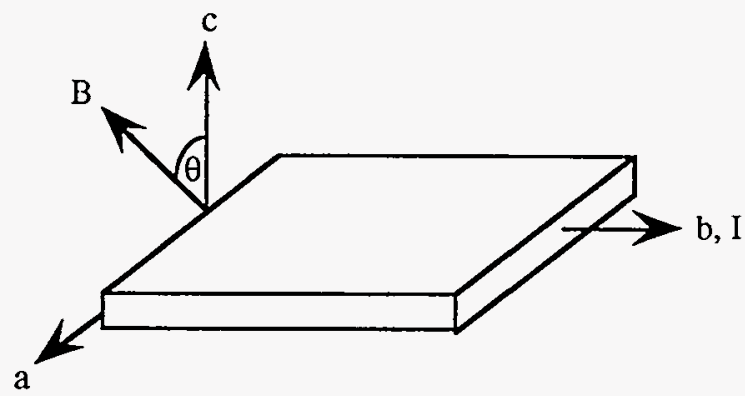

Fig. 1. Definition of axes, current and field direction for an HTS conductor. 


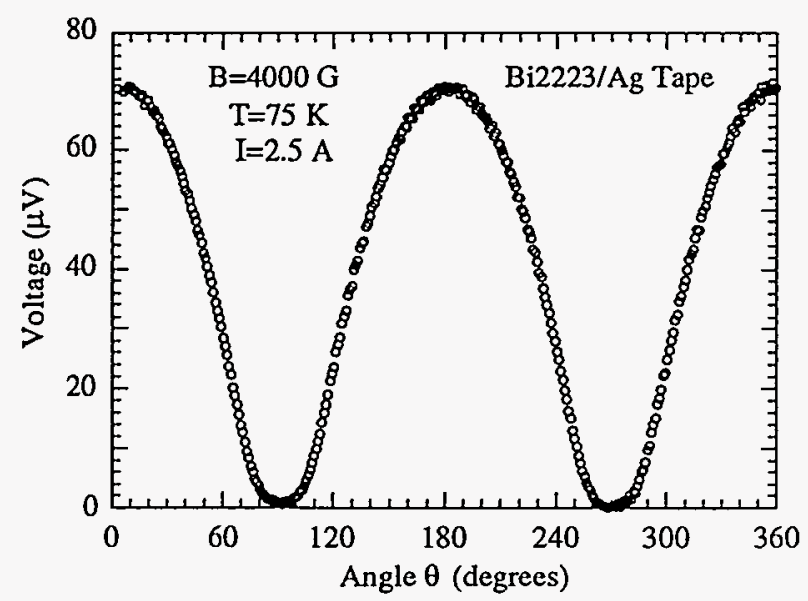

Fig. 2. Voltage drop in a BSCCO sample as a function of external magnetic field angle.

Figure 4 shows the resistance and voltage drop values for the same $10 \mathrm{~cm}$ long thallium sample. The magnetic field is parallel to the $c$ axis and is variable. The resistance of the sample changes by six orders of magnitude from about $10^{-7}$ $\Omega$ to $10^{-1} \Omega$ for a magnetic field which changes between zero and 800 Gauss. The current through the sample was kept constant at $0.3 \mathrm{~A}$ and the temperature was $75 \mathrm{~K}$. The resistance change of six orders of magnitude in a HTS is comparable to the resistance change of a semiconductor, switching between the on and the off state. It appears that the large resistance change can be exploited for some applications. Such applications can be in the form of a variable resistor by changing the magnetic field in a controlled fashion or as a switch. In the case of the switch only two states, one without an external field and one with a large enough external field to saturate the HTS, are necessary.

Thallium based superconductor meander line samples with a total length of superconductor of $1 \mathrm{~m}$ are commercially available[5]. This sample would have a resistance of less than $10^{-6} \Omega$ in zero field and a value of several $\Omega$ in an applied field of close to 1000 Gauss. The voltage drop for

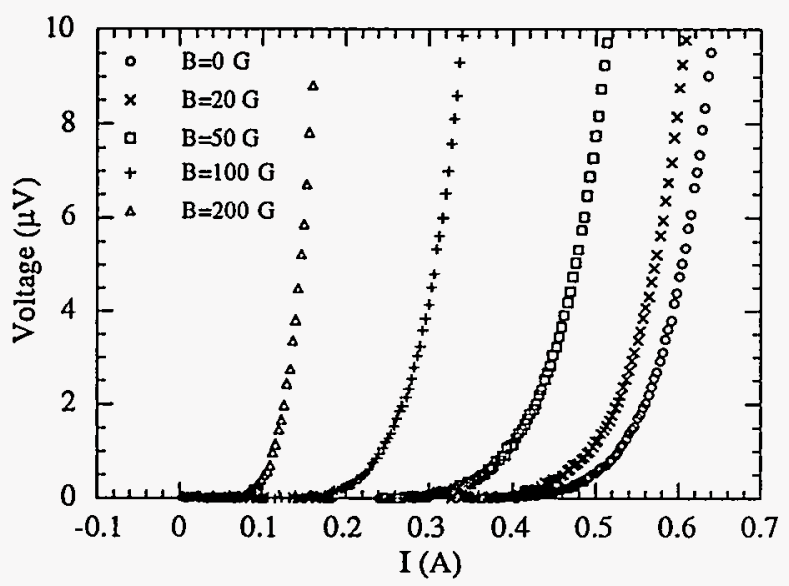

Fig. 3. Voltage-current relationship for a thallium based sample with the external field as a variable.

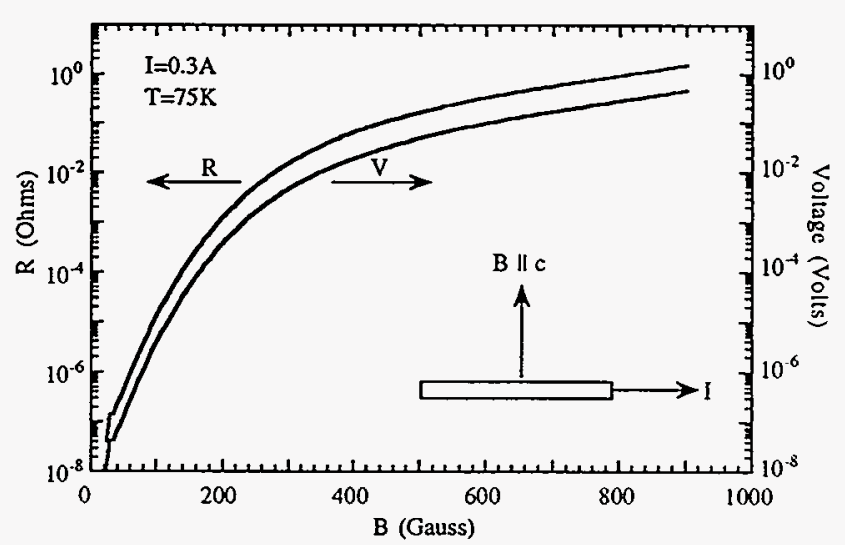

Fig. 4. Voltage and resistance as a function of external magnetic field for a $10 \mathrm{~cm}$ long thallium based HTS.

the high resistance state would be close to $10 \mathrm{~V}$. By increasing the wafer size it can be envisioned that samples with lengths reaching $1000 \mathrm{~m}$ and conductor widths of 0.1 $\mathrm{mm}$ can be manufactured. These lengths and conductor widths are necessary for high voltage and high current nonlinear resistors and switches, required for the electrical power industry.

\section{HEAT TRANSFER CALCULATION}

The application of the HTS material as a variable resistor or switch is limited by the amount of heat which can be removed from the sample, when it goes into the resistive mode. If the HTS material is deposited on a low thermal conductivity substrate such as the Lanthanum Aluminate substrate, which the Thallium 2212 meander line was deposited on, then the effective surface area for heat transfer calculations will be limited to the surface of the superconductor itself. For our meander line the width was $0.006 \mathrm{~cm}$ and the length was $10 \mathrm{~cm}$, resulting in a surface area of $0.06 \mathrm{~cm}^{2}$. As long as the temperature difference between the bath $(77 \mathrm{~K})$ and the surface of the HTS is kept below 8 to $10 \mathrm{~K}$, we can expect nucleate boiling at the surface of the sample. While there is a moderate amount of scatter in the data on nucleate boiling in liquid nitrogen, the data can be summarized by the Kutateladze Correlation[6]. Using the correlation and a curve fit, we can obtain results for the nucleate boiling heat flux in liquid nitrogen at $1 \mathrm{~atm}$ as shown in Fig. 5. Using this curve fit and the above surface area results in a heat removal capacity of $0.4 \mathrm{~W}$ for the meander line with an $8 \mathrm{~K}$ temperature difference between the surface and the bath temperature. If a high conductivity substrate such as sapphire were used, the effective surface area can be increased by a factor of four, because the appropriate surface area would include the area between the meander line segments and the back of the substrate. This would result in a heat removal capacity of about $1.6 \mathrm{~W}$ for the meander line of $25 \mathrm{~mm}^{2}$ surface area. If higher heat removal capacities are desired, the surface area between the superconducting meander line can be increased to provide more surface area for nucleate boiling. 


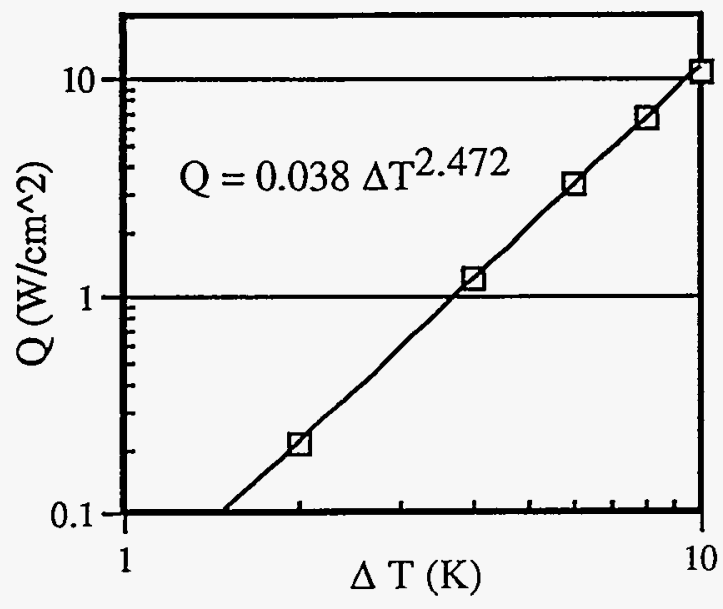

Fig. 5. Nucleate boiling heat flux in liquid nitrogen at 1 atm.

\section{APPLICATIONS}

Use of the field dependent resistor in the form of a variable resistor and switch can be envisioned. Below several examples are given:

1) Variable Dump Resistor: Many superconducting magnets are protected by a dump resistor. In the case of a quench of the superconducting magnet, a circuit breaker interrupts the magnet current and inserts a dump resistor into the circuit. The magnetic energy stored in the magnet is converted into heat in the dump resistor. With a linear dump resistor the current decreases exponentially. With a nonlinear resistor, as can be achieved by changing the external magnetic field of a HTS wire, the resistor can be controlled to increase in value with decreasing current, thus obtaining a constant voltage magnet discharge. In this case, the coil current decreases faster than with a linear resistor, allowing the superconducting coil to heat up less. In Fig. 6 the two curves for the current discharge are shown, one for the linear and one for the nonlinear dump resistor. Also the curves for the coil voltage are given. The assumption was made that the resistance of the quenching magnet is still small and the heating in the magnet is negligible. In a practical application it will be sufficient for the resistor to change in value by a factor of three to four from the initial value at the beginning of the quench. The variable dump resistor has the advantage that the dc breaker can be eliminated in the circuit.

2. Fault Current Limiter: A natural application of the low and high resistance state of a HTS wire is as a fault current limiter. A fault current limiter is a device that reduces the short circuit current in an ac system to a predetermined allowable lower value. During normal operation the HTS wire, installed in each phase of a power system, has no external field applied. The resistance value of the superconducting wire is extremely low. If a fault occurs in the system, the fault current is sensed and the background field for the HTS wire is turned on, which results in a resistance increase in the circuit and in a reduction of the fault current. A simplified circuit diagram of a fault current limiter is given in Fig. 7. Included in the figure is a current sensing

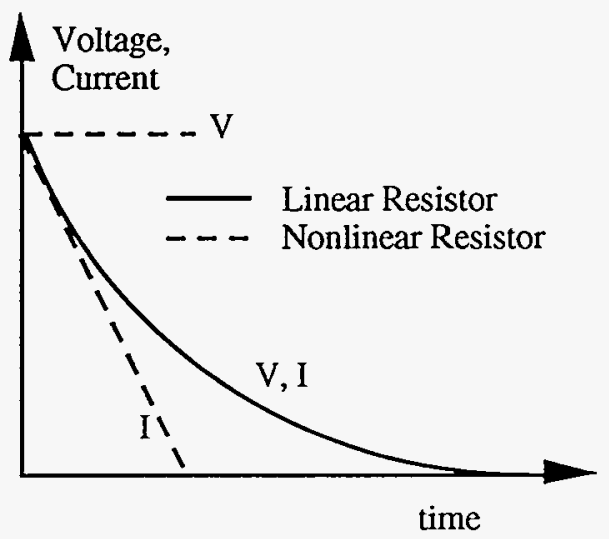

Fig. 6. Magnet discharge voltage and current for a linear and nonlinear dump resistor.

unit which measures the initial current rise of the fault current and triggers the current flow for the background magnet. Controlling the value of the background field adjusts the resistance of the superconducting wire and the fault current level. The adjustable fault current feature is a desirable feature for some electric utilities.

3) Current Rectification: Rectifier circuits, which convert ac power into dc power, can be constructed using HTS wires with a field dependent resistance. Figure 8 shows a singlephase, full-wave rectifier circuit, in which the conventional diodes are replaced by HTS wires, which are subjected to an external magnetic field. Because a set of two conductors will always be either in the low or high resistance state, it is sufficient to have two external fields, with conductors 1 and 2 exposed to one field and conductors 3 and 4 to the other field. If we assume a sinusoidal voltage source and control the two magnetic fields in such a way, that conductors 1 and 2 are in the low resistance state during the time of the positive half wave of the source voltage and conductors 3 and 4 during the negative half sine wave, then we achieve a rectified ac sine wave as the load voltage. The external fields must be controlled in such a way, that one set of conductors is in the

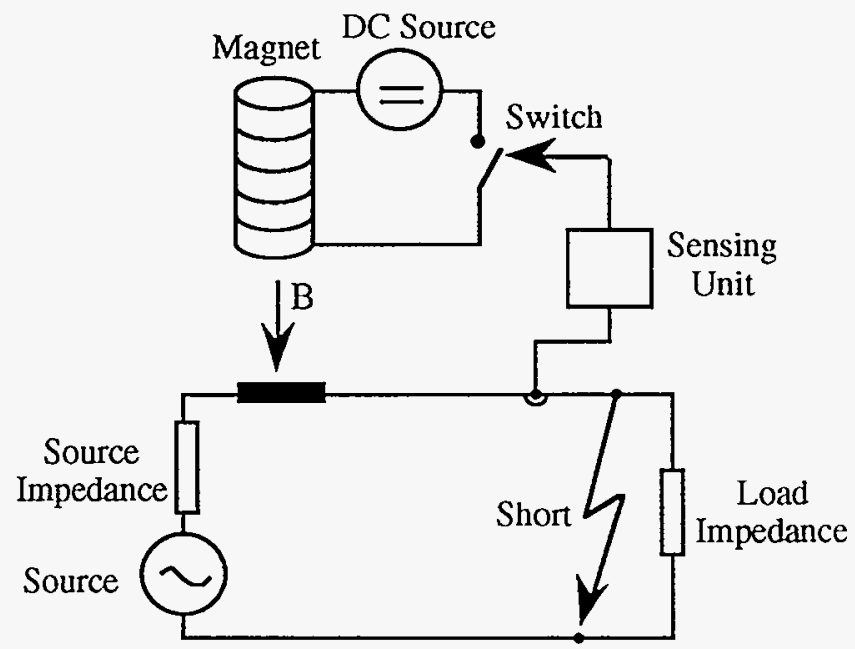

Fig. 7. Fault current limiter using HTS wires. 

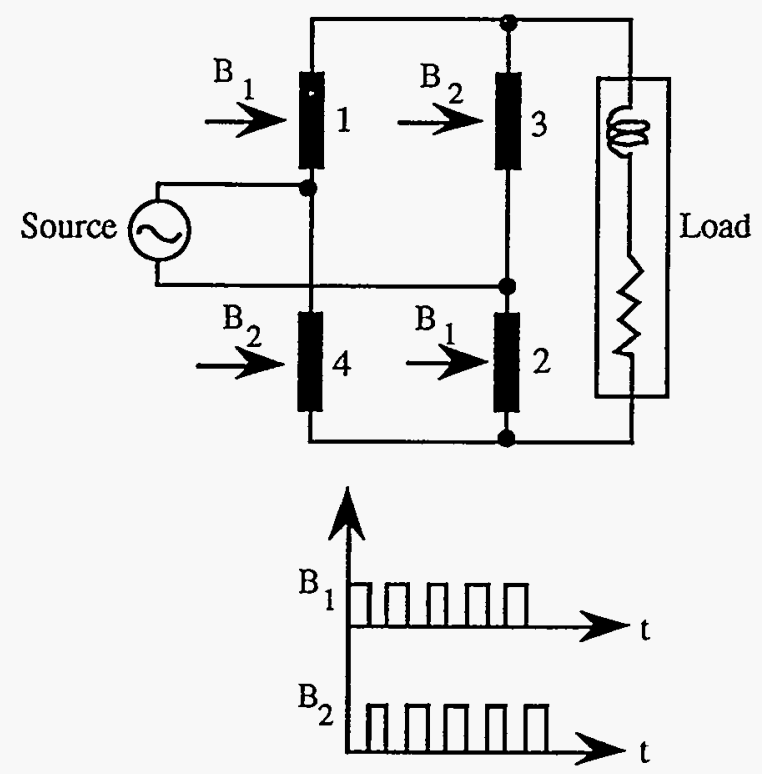

Fig. 8. Single-phase, full-wave bridge rectifier using HTSs as switching elements.

low resistance state with the other set in the high resistance state. Field switching must take place in synchronism with the input voltage frequency. The basic idea as shown for a single-phase circuit can be extended to multiphase circuits. The operation is not limited to rectification, but inversion can also be obtained. If the conductors 1 and 2 are placed in the low resistance state during the negative voltage time periods and the load is inductive, then the average load voltage becomes negative and energy is drawn from the inductive load into the source. It can be summarized that the field dependent HTS conductor shows some characteristics of both a solid state diode and thyristor when applied to converter circuits.

\section{SUMMARY}

Anisotropic HTS conductors show critical current densities and therefore resistance values which are highly dependent on the direction and amplitude of an applied external field. Differences in resistance values by several orders of magnitude can be measured with existing HTS wires. The change in resistance as a function of field can be exploited in designing equipment using the anisotropic superconductors as variable resistors and switches. While results with existing wire samples are limited to small voltage, current and power levels, these results encourage further research.

\section{REFERENCES}

[1] R. H. Ono, L. F. Goodrich, J. A. Beall, M. E. Johansson, and C. D. Reintsema, "Magnetic field dependence of the critical current anisotropy in normal metal- $\mathrm{YBa}_{2} \mathrm{Cu}_{3} \mathrm{O}_{7-\delta}$ thin-film bilayers," Appl. Phys. Lett. 58 (11), pp 1205-1207, March 1991.

[2] Y. Tzeng, C. Cutshaw, T. Roppel, C. Wu, C. W. Tanger, M. Belser, R. Williams, L. Czekala, M. Fernandez, and R. Askew, "High-temperature superconductor opening switch," Appl. Phys Lett. 54 (10), pp 949-951, March 1989.

[3] K. E. Gray, T. Lenihan, and J. Tarczon, "Thin Film Superconducting Switches," IEEE Trans. on Magnetics, MAG-15, pp 175, 1979.

[4] S. Fleshler, M. P. Maley, J. Y. Coulter, X. D. Wu, and Q. Jia, "Transport Properties of $\mathrm{Tl}_{2} \mathrm{Ba}_{2} \mathrm{CaCu}_{2} \mathrm{O}_{8}$ Meander Lines," paper presented at the 1994 Applied Superconductivity Conference.

[5] W. L. Holstein, C. Wilker, D. B. Laubacher, D. W. Face, P. Pang, M. S. Warrington, C. F. Carter, and L. A. Parisi, "Critical current density and resistivity measurements for long patterned lines in $\mathrm{Tl}_{2} \mathrm{Ba}_{2} \mathrm{CaCu}_{2} \mathrm{O}_{8}$ thin films," J. Appl. Phys., vol. 74, pp. 14261430 , July 1993.

[6] E. G. Brentari, P. J. Giarratano and R. V. Smith, "Boiling Heat Transfer for Oxygen, Nitrogen, Hydrogen and Helium," NBS Technical Note No. 317, September 20, 1965.

\section{DISCLAIMER}

This report was prepared as an account of work sponsored by an agency of the United States Government. Neither the United States Government nor any agency thereof, nor any of their employees, makes any warranty, express or implied, or assumes any legal liability or responsibility for the accuracy, completeness, or usefulness of any information, apparatus, product, or process disclosed, or represents that its use would not infringe privately owned rights. Reference herein to any specific commercial product, process, or service by trade name, trademark, manufacturer, or otherwise does not necessarily constitute or imply its endorsement, recommendation, or favoring by the United States Government or any agency thereof. The views and opinions of authors expressed herein do not necessarily state or reflect those of the United States Government or any agency thereof. 